\title{
SITUACIÓN LABORAL DE LA MUJER UNIVERSITARIA EN ANDALUCÍA
}

\author{
María Teresa ARÉVALO QUIJADA \\ María José VÁZQUEZ CUETO
}

\section{INTRODUCCIÓN}

El análisis de la situación laboral de la mujer pasa obviamente por la consideración de múltiples variables, no sólo económicas sino también sociológicas e incluso geográficas. En España la participación femenina en la actividad económica depende, en buena medida, de la situación familiar, y así, el número de hijos, las edades de éstos, el estado civil, el trabajo del cónyuge..., se muestran factores determinantes en la decisión de la mujer de incorporarse o no, y de qué forma, al mercado de trabajo. Aunque en 1997 la proporción de mujeres activas frente a la población femenina es, junto con Italia, una de las más bajas de los países de la OCDE, alrededor del $38 \%$, es un hecho constatado que, en términos generales, el posicionamiento de la mujer en el mercado de trabajo ha mejorado ostensiblemente en los últimos tiempos. Así, y aunque la situación actual está aún muy lejos de lo que sería deseable en una sociedad desarrollada, los avances han sido considerables: La proporción a que aludíamos anteriormente ha aumentado en nueve puntos porcentuales en los últimos veinte años. Este cambio experimentado no ha afectado por igual a todas las regiones ni a todos los segmentos poblacionales femeninos que consideremos.

En Andalucía, la participación femenina, medida a través del número de mujeres que mostraban su deseo de trabajar declarándose activas en las EPAs, se ha visto incrementada por encima del aumento experimentado en otras regiones. Sin embargo, no se ha generado en esta Comunidad el suficiente empleo para absorber tanta demanda, encontrándonos, en consecuencia, con un paro, que podríamos considerar endémico y rígido a la baja, que se encuentra en unos niveles muy superiores a los del resto del territorio Nacional.

En un estudio anterior centrado en la Comunidad Autónoma de Andalucía, para el periodo 1985-1996, hemos detectado que la situación de la mujer en el mercado laboral varía según su edad y su nivel educativo, resultando que la mujer andaluza con estudios universitarios se ve ampliamente favorecida en relación con 
otros colectivos considerados: La proporción de activas se sitúa veinte puntos por encima del total de la población femenina andaluza, colocándose en más de un 75\% a finales de 1996; la proporción de ocupadas se mueve en las bandas de un $45 \%$ a un $55 \%$, treinta puntos por encima del total poblacional femenino. El desempleo sin embargo, afecta a este colectivo de la misma forma que afecta al total, así de cada 100 mujeres activas nos encontramos con una media de 35 paradas. Dado que nuestra Comunidad presenta datos discordantes con el resto Nacional, nos preguntamos cuál es nuestra posición si nos restringimos a este colectivo.

El objeto del presente trabajo es, pues, analizar la evolución de la situación laboral de la mujer andaluza con estudios universitarios y compararla con el conjunto Nacional. Hemos elegido como periodo temporal 1987 a 1996, en primer lugar porque es el periodo de tiempo en el que los cambios se han producido a una mayor velocidad y, en segundo lugar, porque es un periodo lo suficientemente amplio como para recoger aquellos cambios debidos a la coyuntura económica. Los datos se han recogido de las EPAs correspondientes a los terceros trimestres de cada año, ya que no se producen variaciones significativas de un trimestre a otro y es, quizás, el tercer trimestre el que mejor recoge el número de mujeres con estudios universitarios concluidos durante ese año.

\section{ACTIVIDAD, EMPLEO Y PARO}

Aunque como hemos apuntado anteriormente son muchas las variables que podríamos considerar para analizar la evolución de la situación laboral, obviamente las más significativas son todas aquellas relacionadas con el número de mujeres activas, ocupadas y paradas. En las Tablas 1, 2 y 3 presentamos respectivamente, en millares, el número de mujeres activas, ocupadas y paradas con estudios universitarios, en España y en Andalucía, calculando el porcentaje que representa Andalucía frente a España y el incremento habido en el periodo en estudio.

TABLA 1

NÚMERO DE MUJERES CON ESTUDIOS UNIVERSITARIOS ACTIVAS

\begin{tabular}{lrrrrrrrrrrr}
\hline & 1987 & 1988 & 1989 & 1990 & 1991 & 1992 & 1993 & 1994 & 1995 & 1996 & $\Delta$ \\
\hline España & 626,6 & 635,5 & 756,9 & 776,6 & 843 & 855,2 & 916,8 & 1007,7 & 1084,7 & 1218,5 & $94,4 \%$ \\
Andalucía & 92,9 & 98,4 & 111,7 & 117,1 & 134,4 & 156,5 & 173 & 187 & 196,8 & 234,3 & $152,2 \%$ \\
Porcentajes & 14,82 & 15,48 & 14,75 & 15,08 & 15,94 & 18,3 & 18,87 & 18,56 & 18,14 & 19,23 & - \\
\hline
\end{tabular}

Fuente: EPA ( $3^{\circ}$ trimestres)

Elaboración propia. 
TABLA 2

NÚMERO DE MUJERES CON ESTUDIOS UNIVERSITARIOS OCUPADAS

\begin{tabular}{lrrrrrrrrrrr}
\hline & 1987 & 1988 & 1989 & 1990 & 1991 & 1992 & 1993 & 1994 & 1995 & 1996 & $\Delta$ \\
\hline España & 470,8 & 482 & 604,8 & 633,1 & 689,1 & 711,3 & 734,2 & 780 & 841,5 & 951,1 & $102,2 \%$ \\
Andalucía & 62,4 & 63,8 & 76 & 85,8 & 100,9 & 116,1 & 120,6 & 122,4 & 135,4 & 161,4 & $158,6 \%$ \\
Porcentaje & 13,25 & 13,24 & 12,57 & 13,55 & 14,64 & 16,32 & 16,42 & 15,69 & 16,09 & 16,97 & - \\
\hline
\end{tabular}

Fuente: EPA ( $3^{\circ}$ trimestres).

Elaboración propia.

TABLA 3

NÚMERO DE MUJERES CON ESTUDIOS UNIVERSITARIOS PARADAS

\begin{tabular}{lrrrrrrrrrrr}
\hline & 1987 & 1988 & 1989 & 1990 & 1991 & 1992 & 1993 & 1994 & 1995 & 1996 & $\Delta$ \\
\hline España & 155,8 & 153,5 & 152,1 & 143,5 & 153,9 & 143,9 & 182,6 & 227,7 & 243,2 & 267,4 & $71,6 \%$ \\
Andalucía & 30,5 & 34,6 & 35,8 & 31,3 & 33,5 & 40,4 & 52,4 & 64,6 & 61,4 & 73 & $139,3 \%$ \\
Porcentaje & 19,57 & 22,54 & 23,54 & 21,81 & 21,77 & 28,07 & 28,7 & 28,37 & 25,25 & 27,3 & - \\
\hline
\end{tabular}

Fuente: EPA ( $3^{\circ}$ trimestres),

Elaboración propia.

Ohservamos en la Tabla 1 que el dato ha crecido ininterrumpidamente durante todo el periodo en estudio, confirmando así el hecho de que cada vez son más las mujeres que desean entrar en el mercado de trabajo. Ahora bien, en Andalucía el número de activas con estudios superiores ha aumentado en un $152,2 \%$, con un importante aumento en 1996, un 19,05\%, mientras que a nivel Nacional el aumento ha sido sólo del $94,46 \%$, destacando el importante aumento $(19,1 \%)$ experimentado el año 1989. En nuestra Comunidad, por tanto, se ha acelerado la incorporación de la mujer universitaria. Ahora bien, estos datos no son representativos pues los aumentos pueden haberse debido sólo a un mayor número de mujeres con estudios superiores sin que haya supuesto esto una mayor participación laboral.

De la Tabla 2 extraemos que el número de ocupadas en nuestra Comunidad ha crecido en 99 miles, es decir, el empleo ha aumentado un $158,65 \%$, mientras que a nivel Nacional el aumento ha sido del $102,02 \%$, destacando de nuevo el año 1989 con un aumento en el número de mujeres universitarias ocupadas del 25,48\%. Ahora bien, de nuevo nos encontramos que poco es lo que puede decirse de estos datos. Puede haber aumentado más el número de ocupadas con estudios superiores en Andalucía simplemente porque haya aumentado más el número de mujeres con estudios superiores, sin que esto signifique, por tanto, que el empleo se comporte mejor en esta Comunidad que a nivel Nacional. 
En la Tabla 3 vemos que el temible desempleo clava sus duras garras en nues tra Comunidad, resultando que en siete años el número de paradas ¡con estudio universitarios! ha pasado de 30,5 miles a 73 miles, lo que supone una aumento de $139,34 \%$, mientras que a nivel Nacional ha aumentado sólo en un $71,63 \%$. En $\epsilon$ año 1989 el número de paradas disminuye ligeramente a nivel Nacional mientra que en Andalucía continúa aumentando. En 1995 los resultados se invierten, dis minuye el número de paradas en Andalucía y aumentan a nivel Nacional. Ahor bien, de nuevo el dato habrá que leerlo con atención. Puede haber aumentado e número de paradas simplemente porque haya aumentado el número de activas manteniéndose la misma proporción, llegando, incluso, en nuestra Comunidad ser los resultados mejores que a nivel Nacional si en este ámbito geográfico e número de mujeres activas con estudios superiores hubiese decrecido o no hubie se aumentado en la misma forma.

Llama la atención los datos relativos a 1989, año en que la Economía se man tiene en un periodo de fuerte expansión. Este hecho positivo se refleja en el nú mero de activas, ocupadas y paradas a nivel Nacional pero no repercute en Anda lucía. Sin embargo, en 1992, año en que la Economía está en franca desaceleració de su crecimiento, el paro en Andalucía se resiente, aumentando en casi sietı puntos.

En cualquier caso deducimos que el simple dato absoluto es poco representati vo y que para un buen análisis de la situación y poder establecer comparacione es necesario relativizar los datos. Es por ello por lo que acudimos a las tasas es pecíficas de actividad, empleo y paro.

\section{TASAS ESPECÍFICAS DE ACTIVIDAD, EMPLEO Y PARO}

Se define la tasa específica de actividad para mujeres con estudios superiore: como el cociente entre el número de mujeres activas con estudios superiores y e total de la población femenina con estudios superiores.

$$
\text { Tasa de actividad }=\frac{\text { número de mujeres activas con estudios superiores }}{\text { total de la población femenina con estudios superiores }}
$$

Representa el número de mujeres con estudios superiores que manifiestan sı deseo de participar en la actividad laboral.

Se define la tasa específica de empleo para mujeres con estudios superiore: como el cociente entre el número de mujeres ocupadas con estudios superiores $\mathrm{J}$ el total de la población femenina con estudios superiores. 


$$
\text { Tasa de empleo }=\frac{\text { número de mujeres ocupadas con estudios superiores }}{\text { total de la población femenina con estudios superiores }}
$$

Representa el número de mujeres con estudios superiores que están ocupadas.

Se define la tasa específica de paro para mujeres con estudios superiores como el cociente entre el número de mujeres desempleadas con estudios superiores y el número de mujeres activas con estudios superiores.

$$
\text { Tasa de paro }=\frac{\text { número de mujeres paradas con estudios superiores }}{\text { población activa femenina con estudios superiores }}
$$

Representa el número de mujeres que, manifestando su deseo de trabajar, están desocupadas.

En las tablas 4 y 5 presentamos, en porcentajes, estas tasas calculadas respectivamente, para España y para Andalucía.

TABLA 4

TASAS FEMENINAS PARA MUJERES CON ESTUDIOS UNIVERSITARIOS DE ACTIVIDAD, EMPLEO Y PARO NACIONALES

\begin{tabular}{lllllllllll}
\hline & 1987 & 1988 & 1989 & 1990 & 1991 & 1992 & 1993 & 1994 & 1995 & 1996 \\
\hline Actividad & 67,57 & 68,47 & 70,95 & 69,70 & 71,65 & 70,51 & 71,36 & 72,45 & 73,69 & 74,12 \\
Empleo & 50,77 & 51,93 & 56,69 & 56,82 & 58,57 & 58,64 & 57,15 & 56,08 & 57,17 & 57,86 \\
Paro & 24,86 & 24,15 & 20,10 & 18,48 & 18,26 & 16,83 & 19,92 & 22,60 & 22,42 & 21,95 \\
\hline
\end{tabular}

Fuente: EPA ( $3^{\circ}$ trimestres).

Elaboración propia.

TABLA 5

TASAS FEMENINAS PARA MUJERES CON ESTUDIOS UNIVERSITARIOS DE ACTIVIDAD, EMPLEO Y PARO EN ANDALUCÍA

\begin{tabular}{lcccccccccc}
\hline & 1987 & 1988 & 1989 & 1990 & 1991 & 1992 & 1993 & 1994 & 1995 & 1996 \\
\hline Actividad & 69,85 & 70,84 & 67,33 & 70,29 & 72,22 & 73,13 & 72,93 & 72,82 & 75,81 & 75,73 \\
Empleo & 46,92 & 45,93 & 45,81 & 51,5 & 54,22 & 54,25 & 50,84 & 47,66 & 52,16 & 52,17 \\
Paro & 32,83 & 35,16 & 32,05 & 26,73 & 24,92 & 25,81 & 30,29 & 34,55 & 31,20 & 31,16 \\
\hline
\end{tabular}

Fuente: EPA ( $3^{\text {* }}$ trimestres).

Elaboración propia. 


\section{GRÁFICO 1}

TASAS DE ACTIVIDAD, PARO Y EMPLEO PARA LA POBLACIÓN FEMENINA CON ESTUDIOS SUPERIORES EN ESPAÑA

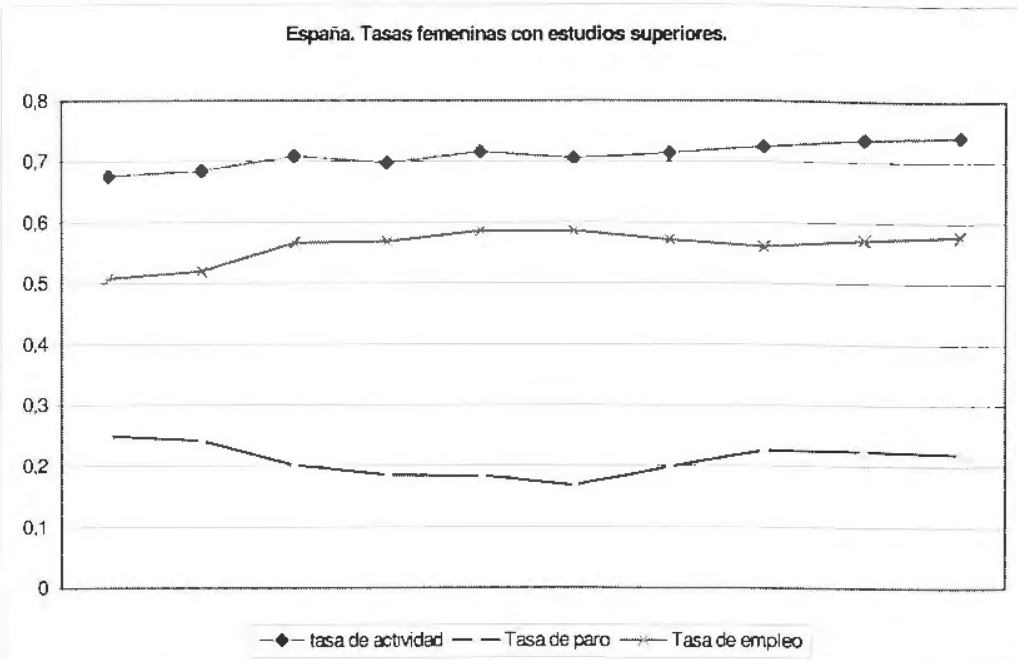

Fuente: EPA ( $3^{\circ}$ trimestres). Elaboración propia.

\section{GRÁFICO 2}

TASAS DE ACTIVIDAD, PARO Y EMPLEO PARA LA POBLACIÓN FEMENINA CON ESTUDIOS SUPERIORES EN ANDALUCÍA

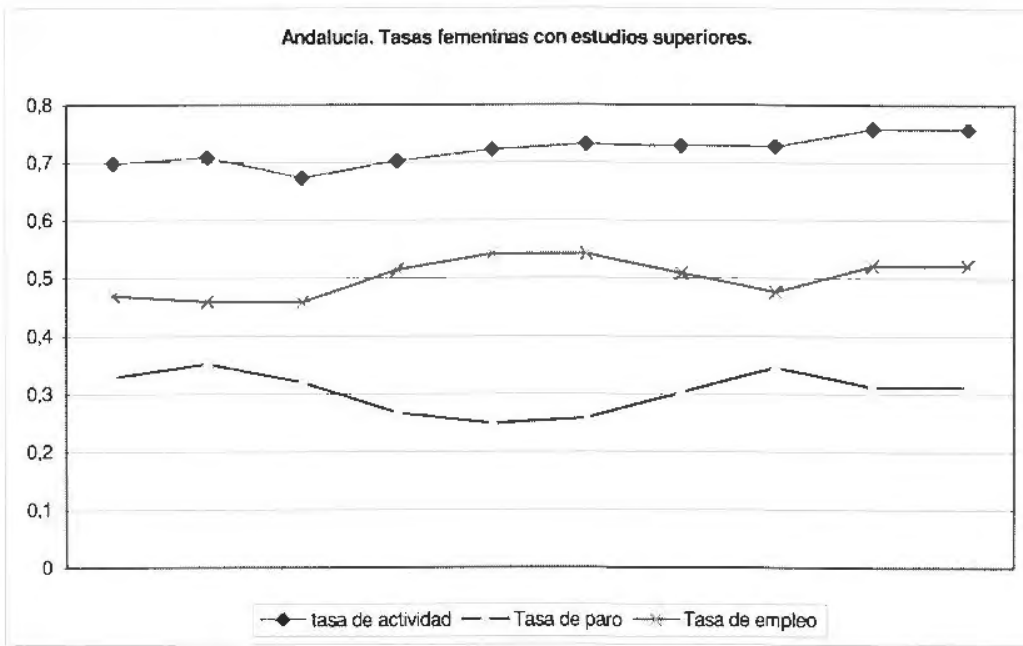

Fuente: EPA ( $3^{\circ}$ trimestres), Elaboración propia. 
Para mejor hacer un estudio pormenorizado y establecer comparaciones entre la situación en Andalucía y el total Nacional vamos a tratar cada una de estas variables en secciones independientes.

\section{ACTIVIDAD}

Lo primero que se desprende del gráfico 3 es que, obviando el dato extraño de 1989, la tasa de actividad de las mujeres andaluzas con estudios universitarios es superior a la correspondiente a nivel Nacional. Ahora bien, en los diez años objeto de estudio las tasas en Andalucía han crecido menos que en España.

TABLA 6

PORCENTAJE DE VARIACIÓN EN LAS TASAS DE ACTIVIDAD

\begin{tabular}{lccc}
\hline & 1987 & 1996 & Variación \\
\hline España & $67,57 \%$ & $74,12 \%$ & $9,69 \%$ \\
Andalucía & $69,85 \%$ & $75,72 \%$ & $8,4 \%$ \\
\hline
\end{tabular}

\section{GRÁFICO 3}

\section{TASAS FEMENINAS DE ACTIVIDAD EN ANDALUCÍA Y EN ESPAÑA}

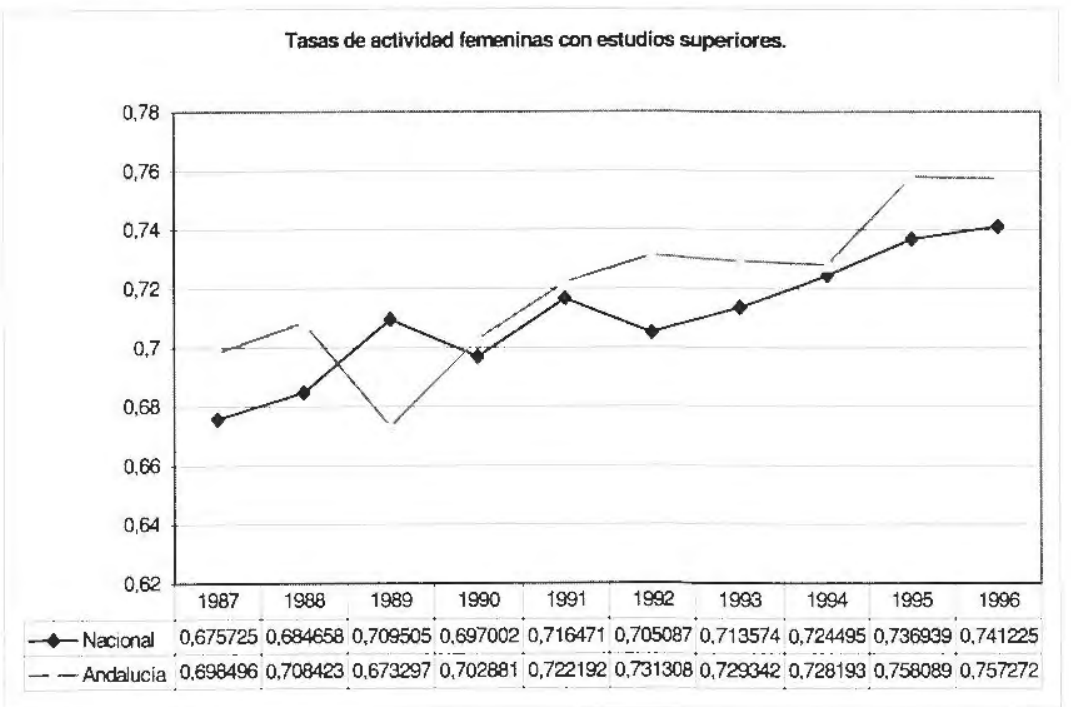

Fuente: EPA ( $3^{\prime \prime}$ trimestres). Elaboración propia. 
De cualquier forma podemos distinguir tres periodos: De 1987 a 1991, époci de crecimiento de la economía, no deja de crecer en Andalucía, resultando ur aumento del 7,26\%, y, sin embargo, oscila en el ámbito nacional.

De 1992 a 1994, época de recesión, la tasa de actividad en Andalucía se com porta de manera inversa a como lo hace a escala nacional. Mientras que en est periodo crece en todo el territorio nacional un 2,75\%, en Andalucía disminuye

De 1994 a 1996 la economía empieza de nuevo a recuperarse y, para la tas: de actividad observamos que a escala nacional aumenta linealmente, con un re sultado de un $2,31 \%$, y en Andalucía expectacularmente, un 3,99\%.

Concluimos que la tasa de actividad no depende de la época de crecimiento c no por la que pase la economía y que en la Comunidad Autónoma de Andalucíc existe un comportamiento diferencial con el resto del territorio nacional.

De todas formas hemos de llamar la atención sobre un hecho que nos parect sorprendente y es que, habida cuenta de que el análisis se restringe a mujeres cor estudios superiores, cuyo coste de oportunidad por no incorporarse al mercadc laboral es grande y sus expectativas de encontrar empleo y bien remunerado sor altas, la tasa de actividad nos indica que en 1996 en España, más de un 25\% de tituladas superiores no muestran deseo de trabajar, es decir, una de cada cuatro Particularizando al caso andaluz la situación es ligeramente mejor dado que e porcentaje de mujeres que se declaran no activas no llega al $25 \%$.

\section{EMPLEO}

Estrechamente relacionado con la participación de la mujer en la actividac económica está el empleo. Numerosos estudios aseguran que altas tasas de emplec conducen a una mayor participación en el futuro.

En el gráfico 4 hemos representado las tasas de empleo andaluzas frente a las nacionales para el colectivo de población en estudio. Lo primero que observamos en este gráfico es que el empleo a nivel Nacional se mantiene siempre por encima del andaluz. Es decir, las mujeres andaluzas con estudios universitarios ven sus esfuerzos compensados en menor medida que las mujeres de otras zonas del territorio nacional. Frente a esto un dato positivo: Durante los años 1987 a 1996 el empleo ha crecido, situándose cerca de un $58 \%$ a nivel Nacional y ligeramente superior a un $52 \%$ a nivel andaluz. La tabla de variación es la que se ve en la página siguiente.

Es un buen comportamiento el del empleo aunque, al igual que ocurría con la tasa de actividad, su aumento se deja sentir menos en nuestra Comunidad. Veamos qué ocurre cuando descomponemos el periodo temporal en la misma forma que lo hicimos anteriormente. 


\section{GRÁFICO 4}

\section{TASAS FEMENINAS DE EMPLEO EN ANDALUCÍA Y EN ESPAÑA}

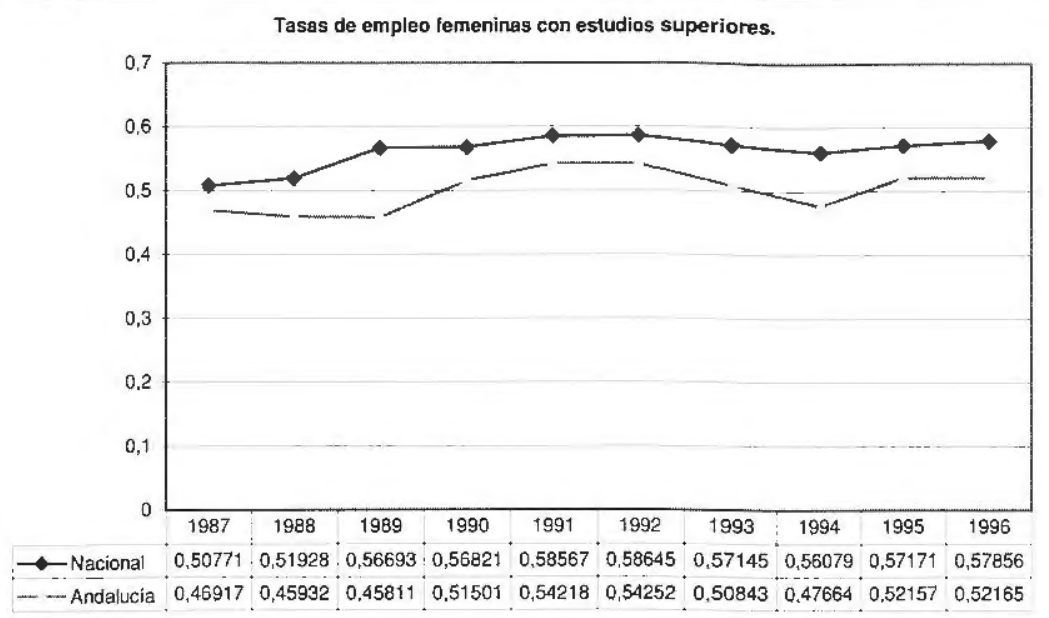

Fuente: EPA (3ำ trimestres). Elaboración propia.

TABLA 7

PORCENTAJES DE VARIACIÓN EN LAS TASAS DE EMPLEO

\begin{tabular}{lccc}
\hline & 1987 & 1996 & Variación \\
\hline España & $50,77 \%$ & $57,86 \%$ & $13,96 \%$ \\
Andalucía & $46,92 \%$ & $52,17 \%$ & $11,19 \%$ \\
\hline
\end{tabular}

De 1987 a 1991 el empleo a nivel Nacional crece continuamente, resultando un aumento del $15,34 \%$. En el ámbito andaluz crece en un $15,56 \%$, en términos globales prácticamente se comporta de igual forma, pero si descomponemos el periodo en dos, observamos que en los años 1987 a 1989 el empleo a nivel nacional crece un $11,66 \%$ mientras que a nivel andaluz disminuye en un $2,34 \%$, la situación de partida, pues, en 1989 es bien distinta. Esto significa que de los años 1989 a 1991 el empleo en las mujeres andaluzas con estudios universitarios se ha comportado mejor.

De 1992 a 1994 las tasas de empleo disminuyen. Ahora bien, mientras sólo lo hace en un 4,36\% en el ámbito nacional, a nivel andaluz lo hace en un 12,15\%. Finalizamos 1994 , pues, con una tasa de empleo nacional del $56,08 \%$ y del $47,66 \%$ andaluz. Las tasas de empleo se han distanciado. 
Entre 1994 y 1996 las tasas de empleo se recuperan, un $3,17 \%$ a escala nacio nal y un $9,46 \%$ a nivel de Andalucía.

Concluimos que la etapa por la que atraviesa la economía tiene una rápida res puesta en la tasa de empleo. Ocurriendo, además, que para el colectivo en estu dio, la tasa de empleo andaluza es más sensible, aumentan más las tasas en bue nas épocas y disminuyen más las tasas en épocas malas. De cualquier forma 1 tasa de empleo andaluza se mantiene cinco puntos, en términos medios, por de bajo de la tasa de empleo nacional.

\section{PARO}

El desempleo es un problema económico y social que afecta a la mayoría di los países de nuestro entorno.

La incidencia del mismo en España es aún mayor, presentando una tasa de parı por encima de la media de los países de la U.E. Además las diferencias regiona les son muy altas, siendo Andalucía la región española con mayor tasa de paro e1 las últimas dos décadas. El paro afecta en mayor medida a la población femenina

La gráfica 5 nos muestra cómo ha evolucionado la tasa de paro para las muje res con estudios superiores tanto en el ámbito nacional como andaluz.

\section{GRÁFICO 5}

\section{TASAS FEMENINAS DE PARO EN ANDALUCÍA Y EN ESPAÑA}

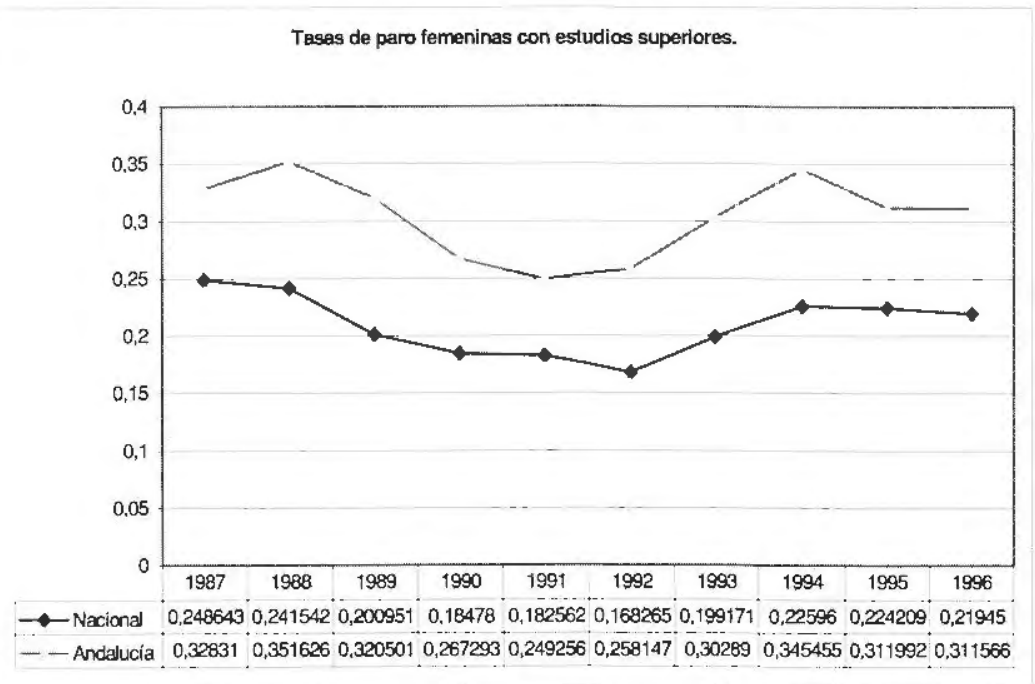

Fuente: EPA (3* trimestres). Elaboración propia. 
Ambas gráficas presentan un perfil idéntico si eliminamos los años 1988 y 1992, manteniéndose una diferencia media en torno a los 9 puntos porcentuales en detrimento de la tasa de paro en Andalucía. La mayor diferencia se alcanza en 1994, 12 puntos porcentuales, año en que la crisis económica es más acusada. La menor diferencia se alcanza en 1991, 6 puntos porcentuales, año en el que podemos situar que acaba la época buena.

La tasa de paro nacional se ha mantenido por debajo del $20 \%$ hasta 1993 , para llegar al 22,5\% en 1994 y mantenerse alrededor de esta cifra en 1995, empezando a mejorar la situación en 1996, con una tasa de paro del 21,9\%.

En el caso de Andalucía se observa un fuerte crecimiento del paro entre los años 1992 y 1994 , en los que la tasa pasa del $25,81 \%$ al $34,54 \%$, lo que supone un aumento de1 33,82\%. En 1995 cae un $9,56 \%$ situándose en un $31 \%$, cifra en la que se mantiene en 1996. Es decir, el paro en Andalucía para el colectivo en estudio se vio muy afectado por la recesión económica y aunque ha mejorado cuando la economía lo ha hecho, aún no ha alcanzado los niveles de los años 90-92, deducimos de este comportamiento que Andalucía es más vulnerable que España, en cuanto al paro se refiere, a la marcha de la economía.

En la tabla 8 hemos recogido la variación entre las tasas de paro a nivel nacional y a nivel andaluz y la variación entre sus diferencias. Hemos intercalado los años 1992 y 1994 para mejor observar su evolución según la etapa por la que atraviesa la economía.

TABLA 8

PORCENTAJES DE VARIACIÓN EN LAS TASAS DE PARO

\begin{tabular}{lccccc}
\hline & 1987 & 1992 & 1994 & 1996 & Variaciones \\
\hline España & $24,86 \%$ & $16,83 \%$ & $22,6 \%$ & $21,95 \%$ & $11,7 \%$ \\
Andalucía & $32,83 \%$ & $25,82 \%$ & $34,55 \%$ & $31,16 \%$ & $5 \%$ \\
Diferencia en puntos & 8 & 9 & 12 & 9,21 & - \\
\hline
\end{tabular}

Para todo el periodo en estudio el paro nacional ha disminuido en un $11,7 \%$ mientras que sólo lo ha hecho en un 5\% a nivel andaluz. Además, las diferencias entre ambas tasas van aumentando hasta 1994, lo que significa que la situación en Andalucía no sólo es peor que en España sino que además se agrava.

De cualquier forma queremos llamar la atención sobre el hecho de que la tasa de paro para el colectivo femenino más cualificado se mantiene por encima del $25 \%$, llegando incluso a alcanzar un $34,5 \%$ el año de mayor crisis en la última década, 1994. 


\section{CONCLUSIONES}

Si medimos la situación laboral de la mujer a través de variables básicas como pueden ser: su deseo de participación (tasa de actividad), su nivel de ocupación (tasa de empleo) y la proporción de mujeres que, queriendo trabajar, no encuentran empleo (tasa de paro), nos encontramos que para el colectivo femenino mejor formado, la situación actual está lejos de lo que debiera ser.

Con datos del tercer trimestre de 1996, en España, de 100 mujeres universitarias sólo 74 se declaran activas, de ellas, sólo 57 están ocupadas.

En Andalucía, de 100 mujeres universitarias, 76 se declaran activas y de ellas sólo 52 están ocupadas.

La inversión en formación es, pues, más rentable, en términos de ocupación y restringiéndonos al colectivo de mujeres que muestran su deseo de participar en el mercado laboral, a nivel Nacional que al nivel de la Comunidad Autónoma de Andalucía.

Estos malos datos que suponen un flash de la situación se ven de alguna medida compensados si analizamos la evolución de los mismos.

Con datos del tercer trimestre de 1987, año de partida de nuestro análisis, de 100 universitarias sólo 68 mostraban su deseo de participación a nivel Nacional y de ellas sólo 51 estaban ocupadas. En Andalucía cerca de 70 universitarias de cada 100 se declaraban activas y de ellas 47 estaban ocupadas.

La tasa de participación en mujeres con estudios universitarios ha aumentado en los diez años de nuestro estudio, habiéndolo hecho más a nivel Nacional que a nivel andaluz.

El número de estas mujeres que han ido directamente a engrosar las listas del paro se ha visto disminuir en este periodo de tiempo, aunque esta disminución ha sido superior en más de un $10 \%$ en todo el territorio Nacional que en la Comunidad Autónoma de Andalucía.

El comportamiento del empleo para este colectivo ha sido relativamente bueno, con aumentos a nivel Nacional y de Andalucía, pero volvemos a encontrarnos que mientras en la primera ha aumentado cerca de un $14 \%$ en la segunda lo ha hecho en un $11 \%$.

Es obvio, por tanto, que la situación en Andalucía 'para nuestro mejor colectivo femenino es peor que en el resto Nacional. Si suponemos que las políticas de empleo, tanto activas como pasivas, se aplican por igual en todo el territorio $\mathrm{Na}$ cional, es evidente que no dan los mismos frutos. Si suponemos, además, que un buen período para la economía del País conlleva un buen período para la economía andaluza, también resulta evidente que su traducción en puestos de trabajo para la mujer cualificada no es la misma. ¿Cuáles son las razones? ¿Son las hipótesis de partida falsas? Partiendo de que este análisis es correcto, la razón del porqué la situación laboral de la mujer universitaria en Andalucía es mala en relación con 
la del mismo colectivo a nivel Nacional habrá que buscarla también en otros ámbitos a los estrictamente económicos. Fundamentalmente en variables socioeconómicas, entre otras en razones culturales en relación con el empresariado andaluz, en el mantenimiento de algunas estructuras sociales arcaicas en la Comunidad Autónoma de Andalucía, relacionadas con la familia y el apego al entorno territorial, que no facilita la movilidad de los factores productivos siguiendo las pautas de la Unión Europea. Si todo ello se suma a los factores de base económica estaremos de acuerdo en que el mercado de trabajo de la mujer en Andalucía presenta una franca debilidad en relación con su entorno inmediato. La inversión en educación y en formación universitaria no es suficiente para trasladar a la mujer universitaria andaluza, a la mujer en general, a los sectores productivos de la economía andaluza ni a sus equipos de dirección. Es necesario un cambio social, un corte radical a muchas actitudes y comportamientos de la sociedad andaluza. La revolución femenina, el aumento de la igualdad de oportunidades para la mujer y su mayor protagonismo social y empresarial están aún por llegar a Andalucía.

\section{BIBLIOGRAFÍA}

ARANGO, J. (1998), «Perfil laboral de las regiones españolas», Economía Aragonesa, julio. «Cambios en la composición del empleo y actividad laboral femenina» (1997), Banco de España, Servicio de Estudios, Documento de Trabajo, $n^{\circ} 9714$.

«Economía, trabajo y sociedad» (1998), Centro de estudios sociológicos.

«La participación laboral femenina y la discriminación salarial en España» (1996), Consejo Económico y Social.

«Panorama socio-laboral de la mujer en España», Gabinete de estudios del CES, años 1995 a 1997.

«Empleo y paro en la Economía Española» (1998), Cuadernos de Información Económica, $\mathrm{n}^{\mathrm{0}} 138$.

«Encuestas de Población Activa», 1987 a 1996, INE.

LABORDA, A. (1998), «Evolución del mercado laboral español en el primer semestre de 1998 y perspectivas a corto plazo», Cuadernos de Información Económica, n⿳ 138, febrero 1998. LJUNGQVIST, L. and SARGENT, T.J. (1998), «The European Unemployment Dilemma», Journal of Political Economy, vol. 106, $\mathrm{n}^{\mathrm{0}} 3$.

MALINVAUD, E. (1979), «Una reconsideración de la teoría del paro», Antoni Bosh.

MONTESINOS, M.D. (1998), «El mercado de trabajo: el espectro de la demanda y la inserción laboral», Economía Aragonesa, julio.

PÉREZ INFANTE, J.I. (1998), «La medición del empleo y el paro en España: Situación y problemas», Cuadernos de Información Económica, no 138, febrero 1998.

TOHARIA, L. (1998), «El mercado de trabajo en España», Mc. Graw Hill.

VÁZQUEZ CUETO, M.J. y otros (1998), «Evolución de la participación femenina en la actividad económica de Andalucía. Período 1985-1995», IEA

VÁZQUEZ CUETO, M.J. y otros (1998), «La situación laboral de la mujer en Andalucía», Fundación Cardenal Espínola. 